\title{
FLEX low resolution spectrometer breadboard activities
}

\author{
A. Altbauer, P. Sandri, D. Lang, L. Pettinato, R. Gabrieli, et \\ al.
}

A. Altbauer, P. Sandri, D. Lang, L. Pettinato, R. Gabrieli, P. Coppo, M. Taccola, Davide Nuzzi, "FLEX low resolution spectrometer breadboard activities," Proc. SPIE 11852, International Conference on Space Optics - ICSO 2020, 1185266 (11 June 2021); doi: 10.1117/12.2600226

SPIE Event: International Conference on Space Optics - ICSO 2021, 2021, Online Only 


\section{International Conference on Space Optics-ICSO 2020}

Virtual Conference

30 March-2 April 2021

Edited by Bruno Cugny, Zoran Sodnik, and Nikos Karafolas
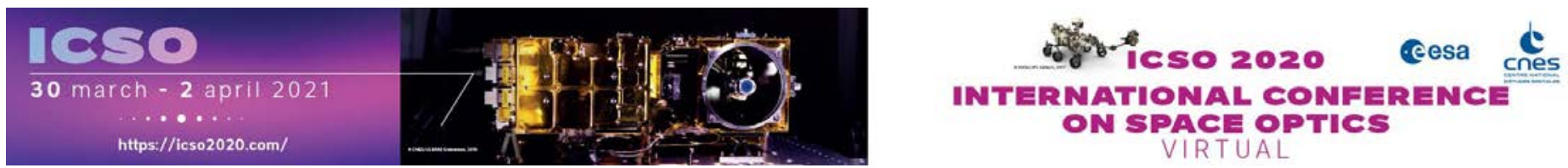

\section{FLEX LOW RESOLUTION SPECTROMETER BREADBOARD ACTIVITIES}

\section{Cesa tropenating ecnes}




\title{
FLEX LOW RESOLUTION SPECTROMETER BREADBOARD ACTIVITIES
}

\author{
A. Altbauer ${ }^{(1)}$, P. Sandri ${ }^{(1)}$, D.A. Lang ${ }^{(1)}$, L. Pettinato ${ }^{(2)}$, R. Gabrieli( ${ }^{(2)}$, P. Coppo $^{(2)}$, M. \\ Taccola ${ }^{(3)}$, D. Nuzzi ${ }^{(4)}$ \\ (1) OHB System AG - Manfred-Fuchs-Str. 1, D-82234 Weßling, Germany \\ (2) Leonardo - SpA Via delle Officine Galileo 1, Campi Bisenzio (FI), 50013, Italy \\ (3) European Space Agency - European Space Research and Technology Center, Noordwijk, \\ The Netherlands \\ (4) Altran Italia SpA Via Guglielmo Marconi 30, 50131 Firenze - Italy (clo Leonardo)
}

Keywords: Spectrometer, Offner, smile, keystone, MTF

\section{INTRODUCTION}

The FLEX Space Segment consists of a single satellite carrying the FLuORescence Imaging Spectrometer (FLORIS) operating in the 500-780 nm spectral band [1]. FLEX will fly in tandem with Copernicus Sentinel-3 working in combination with the Ocean and Land Color Instrument (OLCI) [2] and the Sea and Land Surface Temperature Radiometer (SLSTR) [3,4]. FLEX will provide important additional information regarding the vegetation fluorescence signal and a more accurate atmospheric corrections, which are essential for a quantitative evaluation of the status of health of vegetation. The FLORIS instrument [5] is composed of a High (HRS) and a Low Resolution Spectrometer (LRS), with a spectral sampling and resolution respectively of 0.1 and $0.3 \mathrm{~nm}$ (HRS), and 0.6 and $1.8 \mathrm{~nm}$ (LRS). A flight representative breadboard of the High Resolution Spectrometer was developed by Leonardo and testing results can be found in reference [6]. OHB is responsible of the Low Resolution Spectrometer and another breadboard was designed, manufactured, integrated, aligned and tested at OHB premises. This paper presents the results of the breadboard campaign for the LRS and shows the derived Flight Model (FM) alignment strategy.

\section{OPTICAL LAYOUT OF THE BREADBOARD LRSPE}

The optical layout of the Breadboard of the Low Resolution Spectrometer of FLEX relies on a 1x Magnification Offner Relay. Among the different types of Spectrometers, e.g. the Dyson, the Littrow and the Gärtner, the Offner type with magnification 1, was selected for its relative simple and compact design and for the design-intrinsic correction for coma and for lateral high order aberrations, achieved thanks to the disposition of the three mirrors M1, M2 and M3 with a common center of curvature. To minimize envelope, mass, manufacturing and integration efforts, mirrors M1 and M3 are merged in a unique element presenting a concave spherical surface and the dispersive element, consisting of a blazed holographic grating with density of $500 \mathrm{lines} / \mathrm{mm}$ and working at diffraction order $m=-1$, is located on the convex surface of mirror M2. The grating has a saw-tooth profile generated by holographic recording and is manufactured by ZEISS. Two refractive elements shaped as meniscus and acting as first and last optical element of the spectrometer, provide a further correction of aberrations. The nominal design, shown in Fig. 1, results in a first order effective focal length of $3168.663 \mathrm{~mm}$, works with magnification -0.99 and achieves the theoretical diffraction limit performance in the spectral range $[500 ; 740] \mathrm{nm}$, for a rectangular Field of View with dimensions $\pm 0.042 \mathrm{~mm}$ (slit width in X-direction) times $\pm 22.05 \mathrm{~mm}$ (slit length in Y-direction). The working relative aperture of the spectrometer is F/6.5, realized with a circular aperture stop placed in front of the dispersive element. The achieved spectral resolution results less than $1.8 \mathrm{~nm}$ in the entire spectral range and the Spectral Sampling Interval (SSI) is $0.560 \mathrm{~nm}$ per pixel size of $28 \mu \mathrm{m}$. The nominal design presents a spectral co-registration error (smile) less than $0.3 \mu \mathrm{m}$ and a spatial co-registration error (keystone) less than $0.1 \mu \mathrm{m}$.

The material selection of the opto-mechanical elements was driven by performance and stability requirements, guaranteeing a spectral stability less than $2.8 \mu \mathrm{m}$ in the thermal environment of $22 \pm 1^{\circ} \mathrm{C}$.

The optical layout is presented Figure 1. Figure 2 and Figure 3 report respectively the theoretical spot diagrams and MTF curves at the Nyquist frequency of $17.86 \mathrm{~mm}^{-1}$, for the minimum and maximum working wavelengths. The main geometrical data of the optical elements of spectrometer are reported in Table 1. 


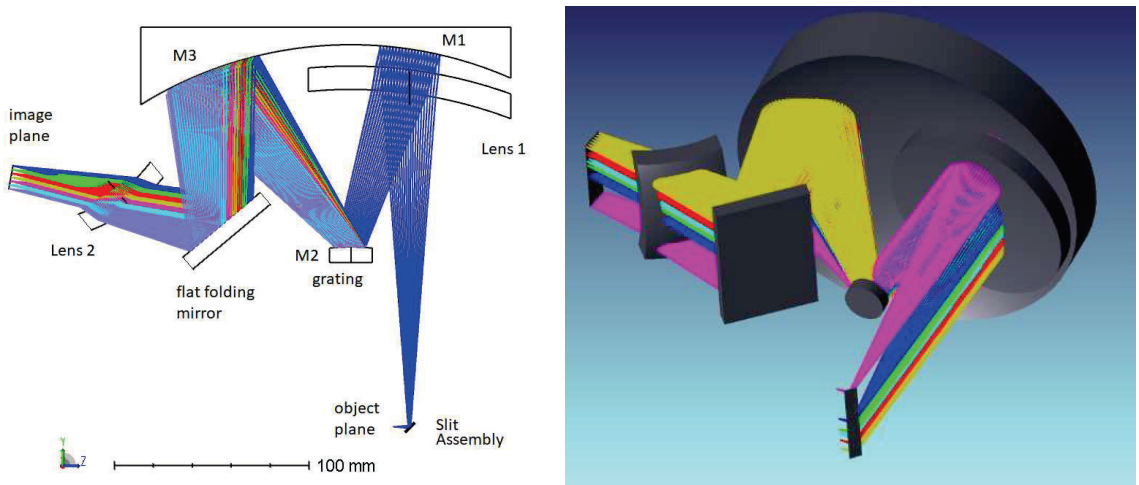

Figure 1: Left/right: 2D/3D optical layout of the Breadboard of the FLEX Low Resolution Spectrometer.

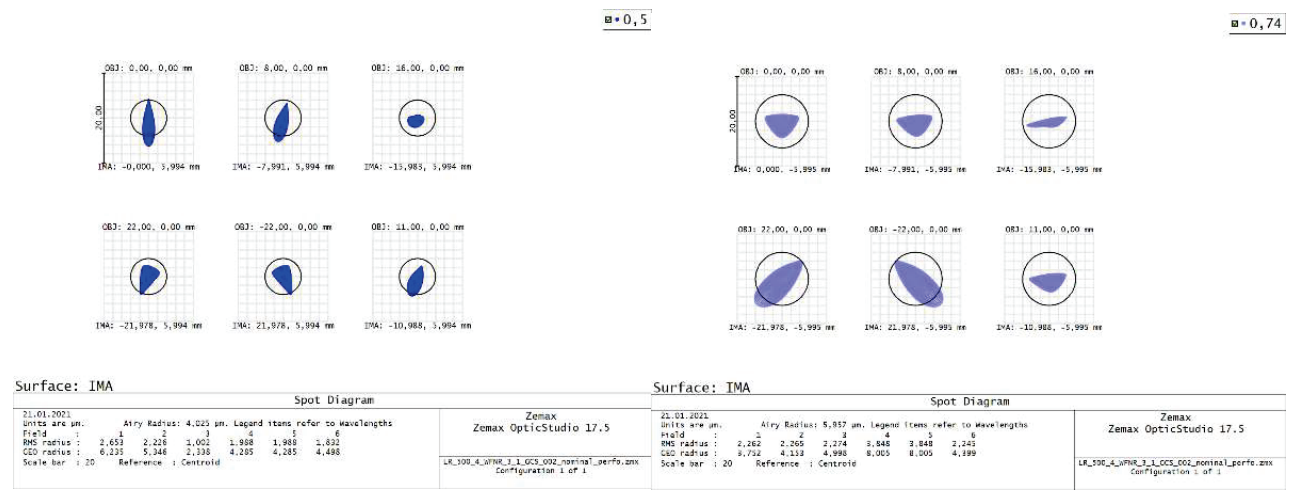

Figure 2: Theoretical spot diagrams of the Breadboard of the FLEX Low Resolution Spectrometer. Left /Right: minimum and maximum working spectral range, compared to the Airy Disk.
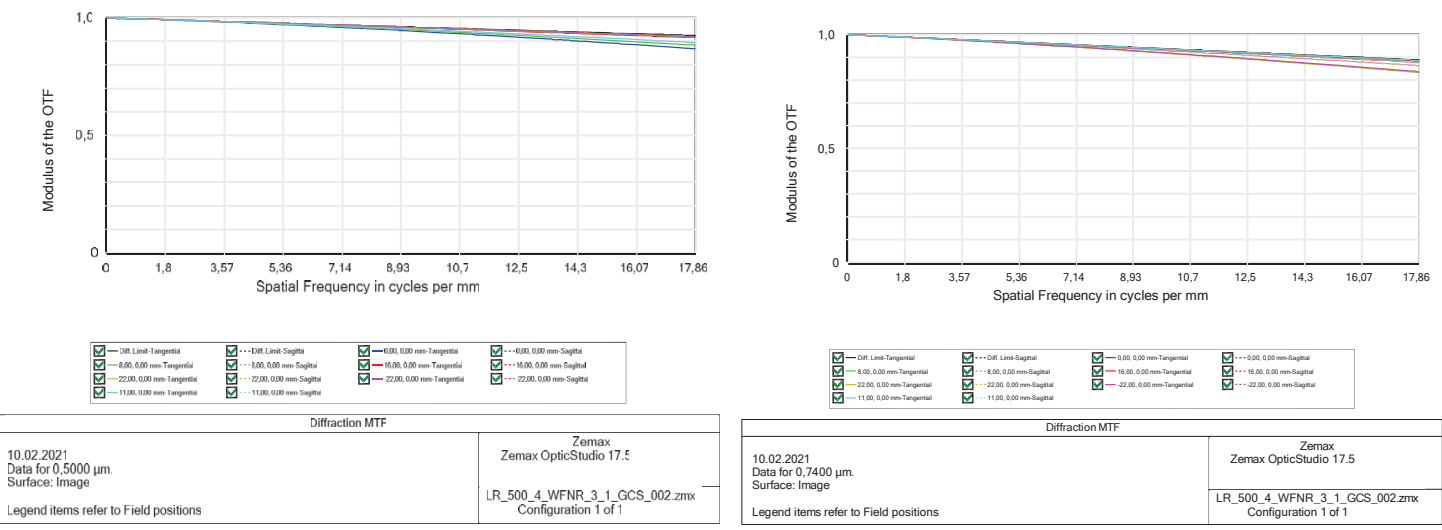

Figure 3: Theoretical MTF curves of the Breadboard of the FLEX Low Resolution Spectrometer. Left /Right: minimum and maximum working spectral range.

Table 1: Main geometry and optical properties of the Breadboard of the LRSPE of the FLEX mission.

\begin{tabular}{|c|c|c|}
\hline Optical element & Material & Optical properties \\
\hline Lens 1 & Schott N-BK7 & $\begin{array}{l}\mathrm{R} 1=231.207 \mathrm{~mm} \text { (spherical convex) } \\
\mathrm{R} 2=242.269 \mathrm{~mm} \text { (spherical concave) } \\
\text { Central thickness }=12 \mathrm{~mm} \\
\text { Antireflective coated in }[500 ; 740] \mathrm{nm} \text {, with reflectivity }<1 \% \\
\text { Surface micro-roughness }<0.3 \mathrm{~nm} \mathrm{rms}\end{array}$ \\
\hline Mirror M1 and M3 & Schott N-BK7 & $\begin{array}{l}\mathrm{R}=242.269 \text { (spherical concave) } \\
\text { Central thickness }=15 \mathrm{~mm} \\
\text { Surface micro roughness }<0.3 \mathrm{~nm} \mathrm{rms} \\
\text { Protected aluminum coated } \\
\text { Back side flat and aluminum coated for thermal reasons }\end{array}$ \\
\hline M2 - grating & Fused silica & $\begin{array}{l}\mathrm{R}=100.165 \text { (spherical convex) } \\
\text { Central thickness }=10 \mathrm{~mm}\end{array}$ \\
\hline
\end{tabular}




\begin{tabular}{|c|c|c|}
\hline & & $\begin{array}{l}\text { Holographic grating density } 500 \text { lines } / \mathrm{mm} \\
\text { Equivalent micro-roughness }<2.0 \mathrm{~nm} \mathrm{rms} \\
\text { Application order } \mathrm{m}=-1 \\
\text { Efficiency in the application order }>60 \% \\
\text { Efficiency of order } \neq 1<10 \% \\
\text { Polarization Sensitivity }<20 \% \\
\text { Bare aluminum coated }\end{array}$ \\
\hline Lens 2 & Schott N-BK7 & $\begin{array}{l}\mathrm{R} 1=87.036 \mathrm{~mm} \text { (spherical convex) } \\
\mathrm{R} 2=77.633 \mathrm{~mm} \text { (spherical concave) } \\
\text { Central thickness }=10 \mathrm{~mm} \\
\text { Surface micro-roughness }<0.3 \mathrm{~nm} \mathrm{rms} \\
\text { Antireflective coated in }[500 ; 740] \mathrm{nm}, \text { with reflectivity }<1 \%\end{array}$ \\
\hline
\end{tabular}

\section{ALIGNMENT CONCEPT TESTED IN BB}

The fine alignment of an optical instrument takes very often benefit of wave front error measurements performed inside an interferometric cavity. For a space instrument, these measurements take place in an optical laboratory in an ISO 5 clean room to guarantee that the needed level of particulate and molecular contamination is preserved. The alignment strategy of the LRSPE is based on the results of the tolerance analysis, which has a two-fold aim. First, to identify the allowed manufacturing and integration tolerances, second to identify a minimum set of adjustments, which allow compensating the manufacturing and integration errors, guaranteeing the achievement of the required instrument optical performances. These compensators can be inside the instrument, as the position of one or more optical elements. In the LRSPE the clocking of grating and the in plane translations of the unique mirror $\mathrm{M} 1+\mathrm{M} 3$, with reference to Figure 2, are used as internal compensators.

The GSE external to the instrument, as for example the position and orientation of the FISLI (SLIT simulator) or the service detector (simulation the Focal Plane) need to be aligned as well. For each compensator a maximum needed travel range and a minimum step size is identified through the tolerance analysis.

The instrument internal alignment strategy for the BB consists in the identification of the optimal position of the mirror M1+M3 in the LRSPE Housing guaranteeing the compliance for the WFE, which is the first image quality parameter to be measured. After the successful position optimization of the mirror M1+M3, the FISLI was installed and the Wave Front Error (WFE) and Modulation Transfer Function (MTF) for all field points and all wavelengths was measured.

Finally, an auxiliary camera, precisely aligned on the image plane, enabled minimization of the keystone, achieved by clocking the grating groves along the optical axis wrt the FISLI.

This alignment strategy resulted successful in the FLEX LRSPE BB campaign, as shown in following Sections.

\section{RESULTS OF THE BB CAMPAIGN}

\subsection{WFE \& MTF}

The instrumental WFE and the demanding MTF were measured inside the operative spectral range from 500 to $740 \mathrm{~nm}$. The measurements were performed by means of a Zygo interferometer working at 632.8nm and of OGSE called "FISLI" in combination with a Shack-Hartmann wavefront sensor.

As a first step, the Zygo interferometer allowing the measurement of the WFE in double pass of the LRSPE at $632.8 \mathrm{~nm}$ was aligned wrt the spectrometer in the position representing the SLIT center (axial). After the light source and measurement device was in place, the back cavity mirror was positioned in the image plane and aligned to enable the WFE measurement (Figure 4). The marginal SLIT positions were repositioned and measured as well. Using these 3 measurements and a sensitivity matrix established in the frame of the tolerance analysis, a fine alignment of the in-plane position of the mirror $\mathrm{M} 1+\mathrm{M} 3$ mirror wrt the grating is performed aiming to minimize the WFE value.

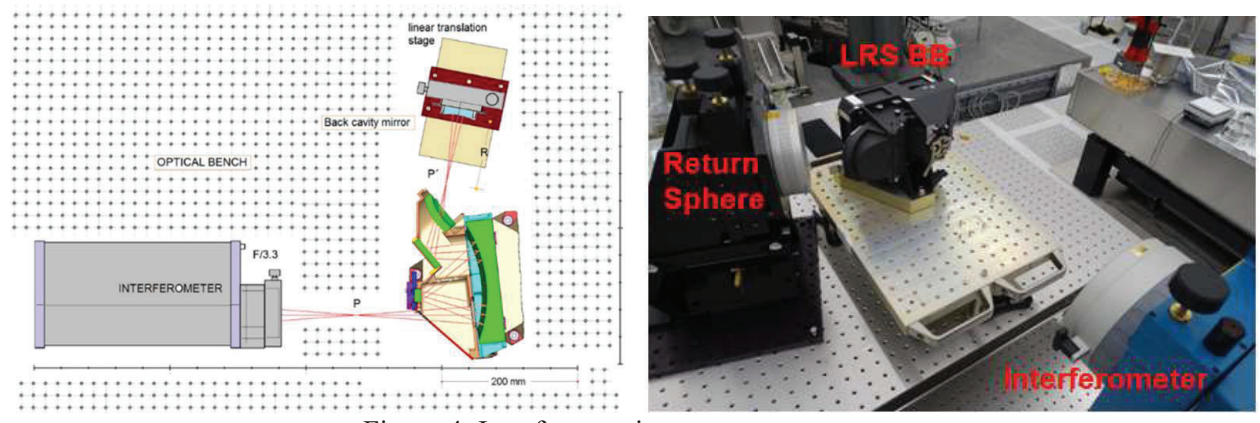

Figure 4: Interferometric measurement set-up. 
After the interferometric measurement, a Shack-Hartmann wavefront sensor (SHS) and the FISLI allowed the measurement of the WFE and of the MTF in single pass at wavelengths different from the $632.8 \mathrm{~nm}$ (Figure 5). For these measurements the FISLI was directly positioned at the input conjugate of the LRSPE, considering the acceptable deviations announced in the tolerance analysis. For the measurements the SHS was aligned in the object plane individually for all field points and all wavelengths. The initial measurements were performed at $632.8 \mathrm{~nm}$ to ensure a proper SHS measurement. Results are presented in the Table 2 and Table 3. Please note: The presence of an additional mirror in the set-up for the interferometer is the reason for the opposite color code in the WFE maps and the sign of the astigmatism.

Table 2: WFE measurements - double pass vs. single pass
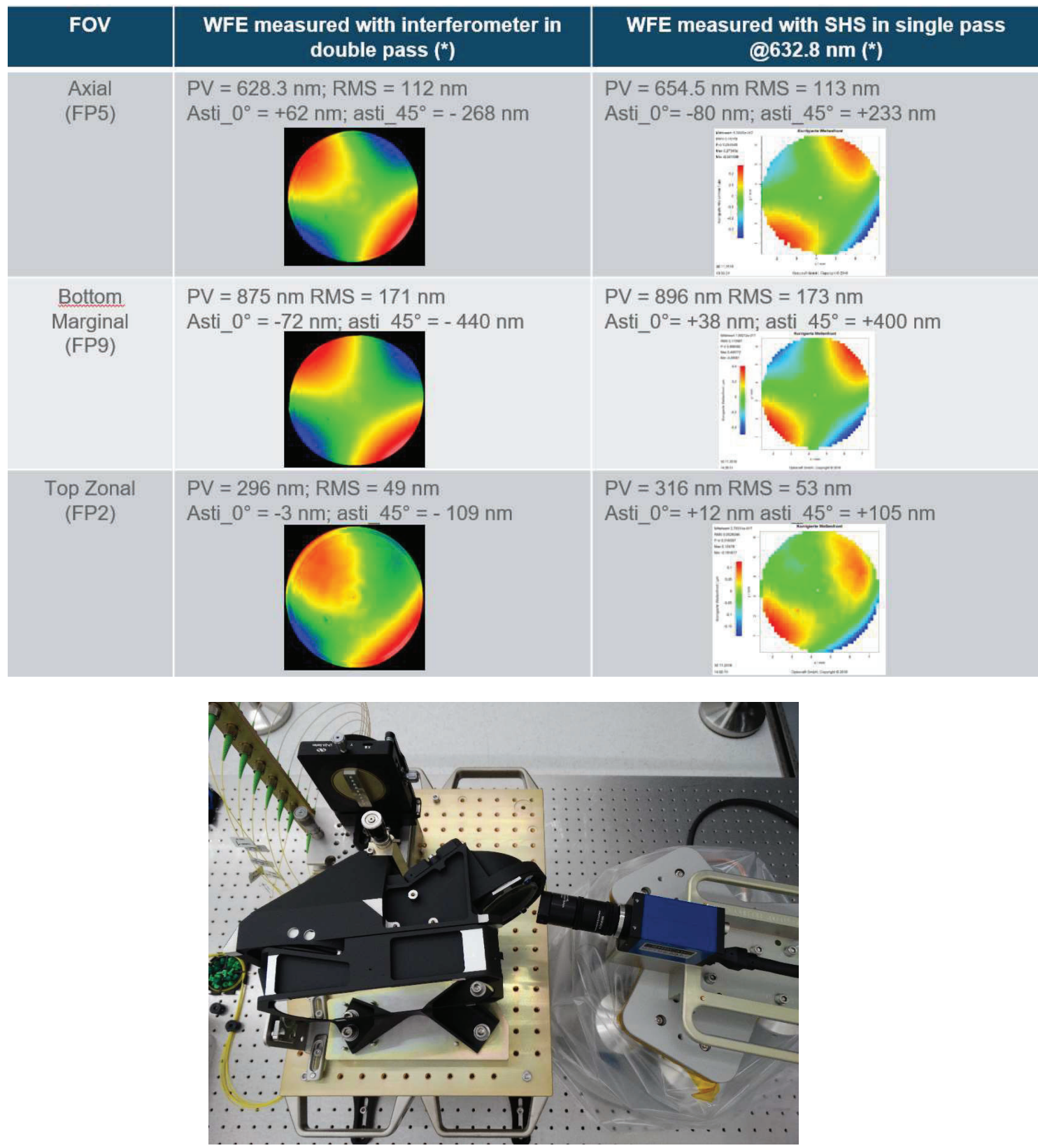

Figure 5: SHS measurement set-up (LRSPE in the center, SHS on the right, FISLI at the top) 
Table 3: MTF - double pass vs. single pass, MTF in single pass is evaluated with Zemax and the measured Zernike values.

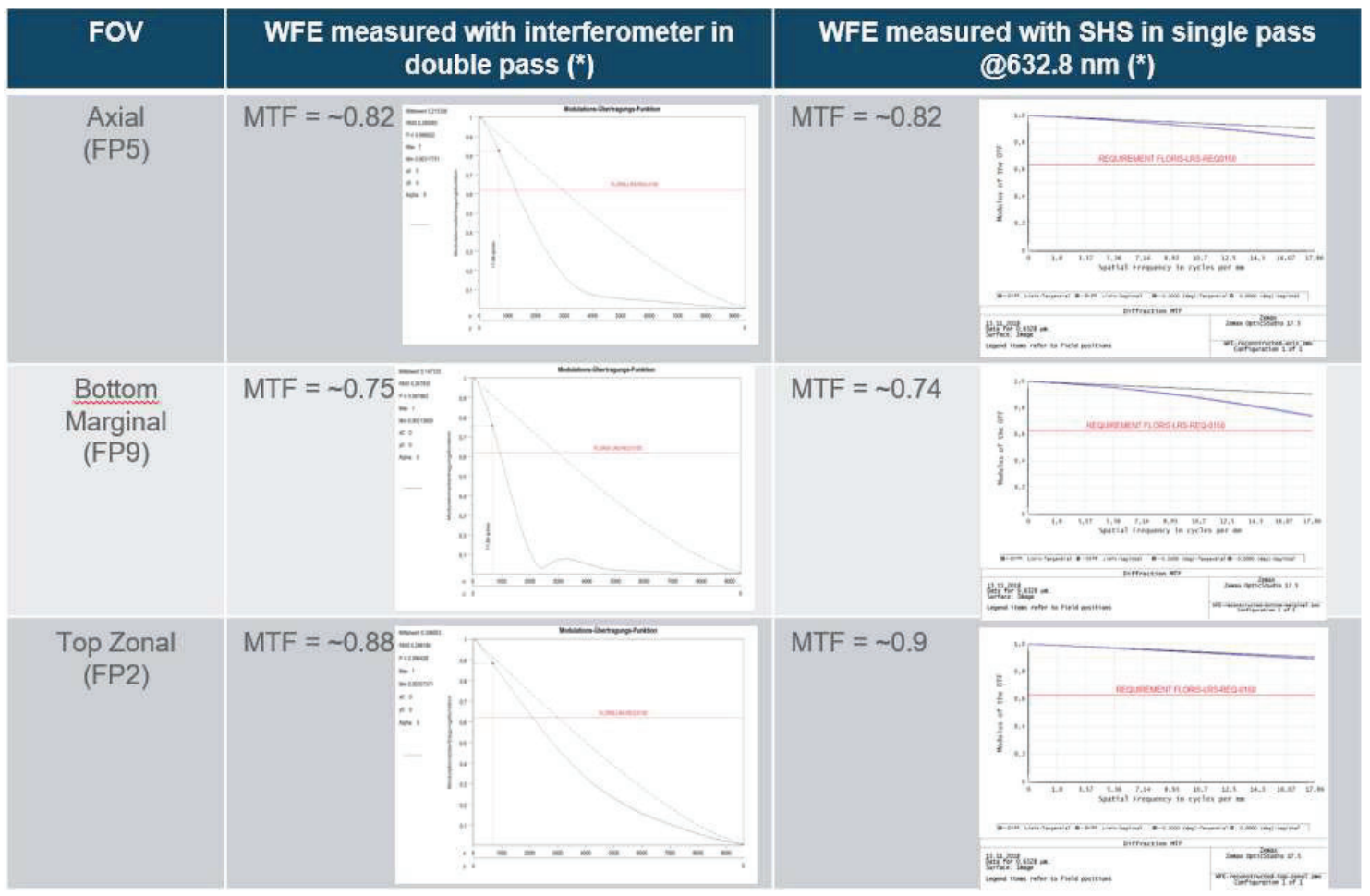

\subsection{Smile \& Keystone}

A special challenge achieved from technical point of view were the tight requirements on the smile and on the keystone, $5.0 \mu \mathrm{m}$ for keystone and $15 \mu \mathrm{m}$ for smile. Those parameters were measured using a service camera precisely aligned on the image plane and the FISLI.

The FISLI was already positioned correctly in the object plane for the SHS measurements (Figure 7). The service detector was aligned in several steps. First the service camera along the optical axis Z to identify the correct back focal distance at axial FoV at $632.8 \mathrm{~nm}$. Next step was the tilt alignment in Y axis using all other field points and finally the tilt alignment in $\mathrm{X}$ axis using all other wavelengths. To make it easily possible the pivot point of the hexapod was set to the center of the CCD detector.

After the successful alignment of the service detector, the clock alignment of the grating or keystone optimization was performed.

Using the service camera flat field measurements and the FISLI characterization results the smile, the keystone, the magnification, the spectral sampling interval (SSI) could be verified successfully within requirements.
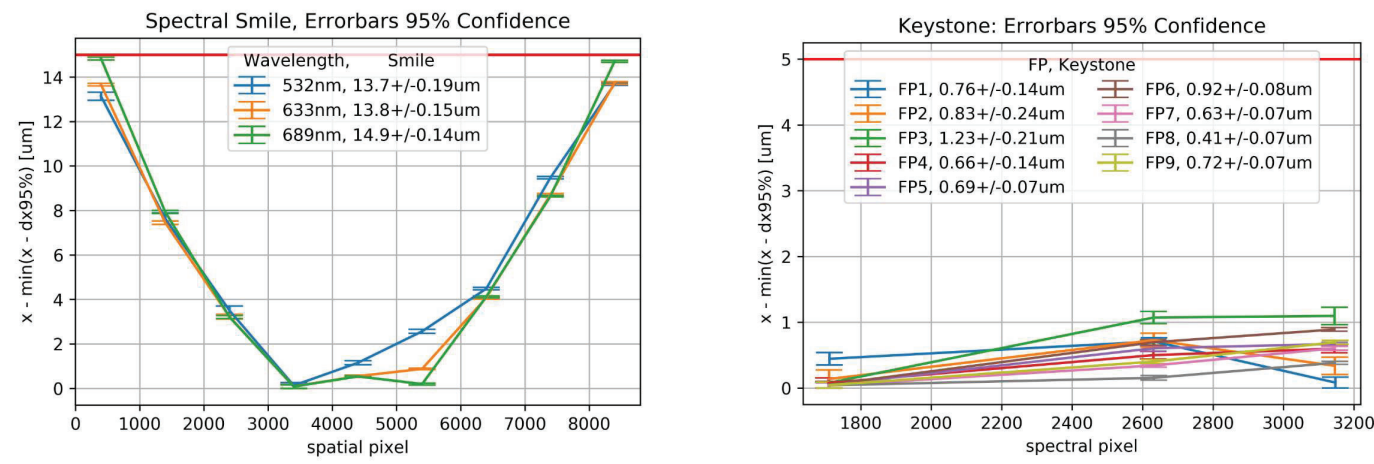

Figure 6: Smile and Keystone measurement results 


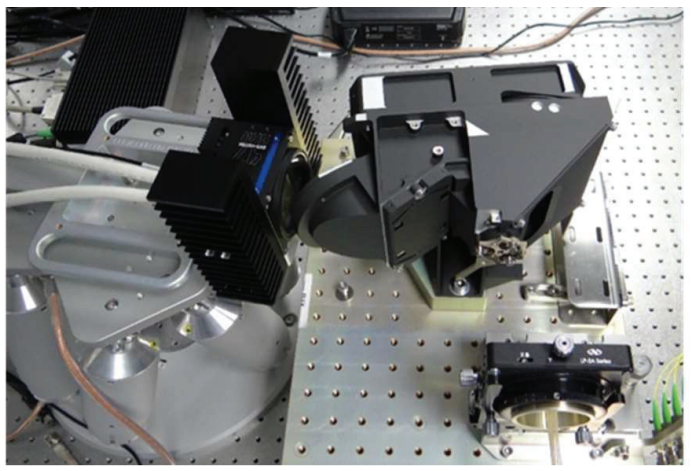

Figure 7: Service Detector measurement set-up

\subsection{Polarization sensitivity}

After the successful validation of the optical performance parameters a polarizer was inserted between FISLI and the LRSPE to enable polarization sensitivity measurements. In this set-up the polarizer was rotatable to measure s- and p-polarization at the LRSPE input and output. Results are presented in Figure 8.

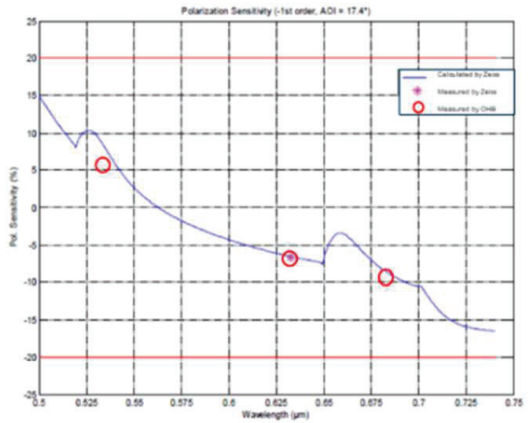

Figure 8: Polarization sensitivity measurement results

Table 4: Summary of measurements.

\begin{tabular}{|l|l|l|l|l|l|l|}
\hline & & WFE & MTF-ALS & Smile & keystone & Pol. Sens. \\
\hline & & & $\mathrm{F}_{\text {Nyq }}=17.86 \mathrm{~mm}^{-1}$ & & & \\
\hline $\begin{array}{l}\text { FM LRSPE } \\
\text { Req. }\end{array}$ & BOL & $350 \mathrm{~nm}$ & 0.65 & $6.3 \mu \mathrm{m}$ & $5.0 \mu \mathrm{m}$ & $18 \%$ \\
\hline & EOL & $380 \mathrm{~nm}$ & 0.63 & $15 \mathrm{um}$ & $5.5 \mu \mathrm{m}$ & $22 \%$ \\
\hline $\begin{array}{l}\text { Bread-Board } \\
\text { LRSPE }\end{array}$ & BOL & $173 \mathrm{~nm}$ & $\begin{array}{l}0.82 \text { on axis } \\
0.74 \text { bottom marg. }\end{array}$ & $14.9 \mu \mathrm{m}$ & $1 \mu \mathrm{m}$ & $<15 \%$ \\
\hline $\begin{array}{l}\text { FM system } \\
\text { performance }\end{array}$ & EOL & $420 \mathrm{~nm}$ & $\begin{array}{l}0.043 \\
\text { SR=1.85 nm }\end{array}$ & $\begin{array}{l}16 \mathrm{um} \\
=0.57 \text { SSI }\end{array}$ & $\begin{array}{l}10 \mathrm{um} \\
=0.12 \mathrm{SSD}\end{array}$ & $\leq 1 \%$ \\
\hline $\begin{array}{l}\text { FM system } \\
\text { requirement }\end{array}$ & EOL & - & SR $\leq 2 \mathrm{~nm}$ & $\leq 1 \mathrm{SSI}$ (goal) & $\leq 0.15 \mathrm{SSD}$ & $\leq 2 \%$ (DOP=50\%) \\
\hline
\end{tabular}

SSI=Spectral Sampling Interval

$\mathrm{SSD}=$ Spatial Sampling Distance

$\mathrm{DOP}=$ Degree Of Polarization (of input radiation)

Fnyq= Nyquist frequency

\section{OPTICAL LAYOUT OF THE FM LRSPE}

Once we entered the C/D Phases of the Program, the optical design was reviewed in order to cover a working spectral range extending up to $758 \mathrm{~nm}$ and in order to fulfill tighter requirements for MTF both along and across track, Spectral Sampling Interval (SSI), stray-light and spectral resolution (SR). Furthermore, the need to withstand high mechanical loads pushed the opto-mechanical design to a light-weighted metal M1/M2 mirror concept. Therefore for the flight spectrometer we also faced manufacturing aspects not encountered in the BB campaign, as for example the stress induced WFE generated by the tightening of the screws of the M1/M2 interfaces and the mid-spatial frequencies manufacturing error generated during the Single-Point-DiamondTurning process and mainly corrected by the application of the Ion-Beam-Figuring process. 
The optical layout is shown in Figure 9, the most noticeable change is that the first lens is not present anymore. The design reaches diffraction limited performances with a simplified layout, with a grating density of 495 lines $/ \mathrm{mm}$ in order to meet a SSI centered to the value of $0.6 \pm 0.006 \mathrm{~nm}$ per $28 \mu \mathrm{m}$ pixel size.
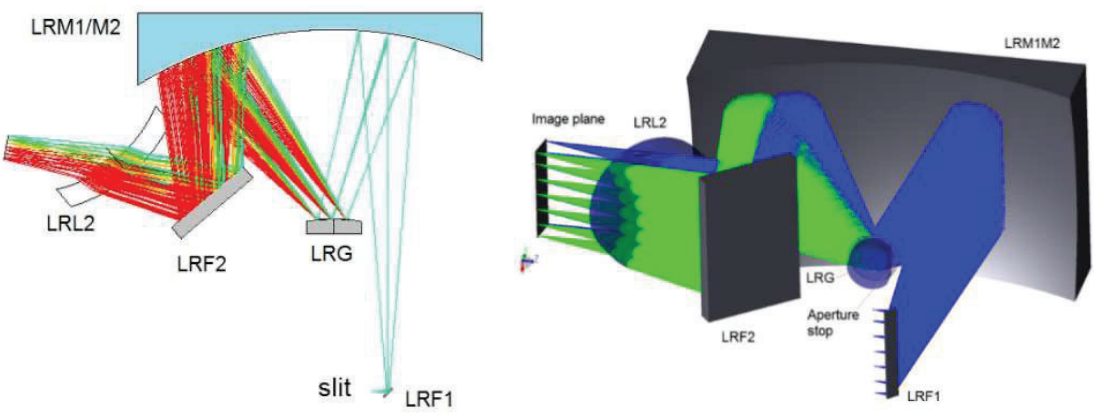

Figure 9: Left/right: 2D/3D optical layout of the FM FLEX Low Resolution Spectrometer.

\section{UPDATED ALIGNMENT CONCEPT FOR FM}

The tightening of the performance requirements for the flight instrument stimulated a review of the alignment strategy, aiming to achieve a method that guarantees the optimization of the WFE and the minimization of values of the co-registration parameters, keystone and smile, whose measurements are planned after the WFE measurement. The intention is to avoid recursive alignment processes.

To identify a suitable criterion that could be implemented in the AIT phases, an analysis was performed on the population of the Monte Carlo Trails generated for the Tolerance Analysis, focusing attention on following parameters:

- $\quad W F E_{r m s}$ for axial FoV

- $\left.\quad|| Z_{6}^{S t}\right|_{0}-\left|Z_{5}^{S t}\right|_{0} \mid$

- $\quad W F E_{r m s}$ for marginal FoV

- $\left.\quad|| Z_{6}^{S t}\right|_{-22}-\left|Z_{5}^{S t}\right|_{+22} \mid$

being $\left|Z_{5,6}^{S t}\right|_{-22}$ the absolute value of the $Z_{5}$ and $Z_{6}$ standard Zernike coefficients for the FoV of $-22 \mathrm{~mm}$. These two standard Zernike coefficients, defined as $Z_{5}=\sqrt{6} \rho^{2} \sin (2 \theta), Z_{6}=\sqrt{6} \rho^{2} \cos (2 \theta)$, represent respectively the primary astigmatism at $45^{\circ}$ and at $0^{\circ}$ (see [7]).

The above mentioned success criteria together with a Sensitivity Matrix of the first 37 Zernike polynomials for rigid body motions, is the Structure of the AIT procedure for the alignment of the flight LRSPE.

\section{FISLI (FIBER SLIT \& ILLUMINATION) OGSE}

The FISLI is simulating the function of an optical slit and simultaneously launching monochromatic light via its optical fibers with the required f-number. The exit facets of the single mode fibers where placed along the hypothetical spectrometer slit (spatial direction) corresponding to 9 field points (Figure 10).

For optical performance measurements of the Low Resolution Spectrometer the FISLI is placed exactly within the slit plane.

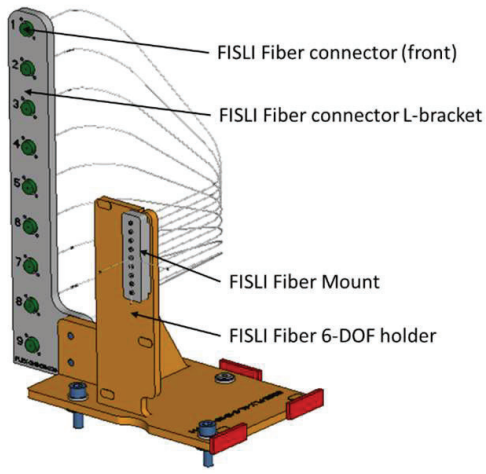

Figure 10: Fibre-based slit assembly FISLI - conceptual PFM design 
The FISLI Fiber Mount was used for BB campaign and will be used for the upcoming FM campaign. Due to the extended wavelength range in FM, an update of the FISLI OGSE for FM is necessary. The BB-refurbished version, the three laser wavelength $(532 \mathrm{~nm} \& 633 \mathrm{~nm} \& 689 \mathrm{~nm})$ were combined via wavelength division multiplexing. This means that all three different wavelengths could be guided to the nine output ports simultaneously. As an illustration, $3 \times 9$ (27) spots on the service detector CCD are highlighted at the same time. This allows one shot measurements to characterize the FLEX spectrometer and to determine aberration errors (such as "smile") (Figure 11).

BB-REF-Version

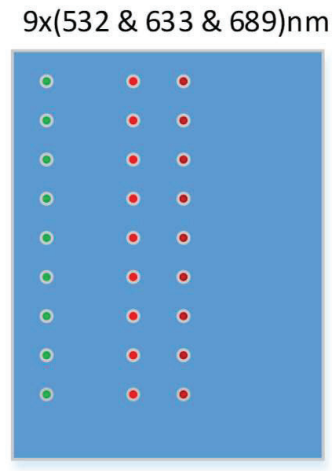

FM-Version

\section{$9 x(532 \& 633 \& 689 \& 703 \& 730) \mathrm{nm}$}

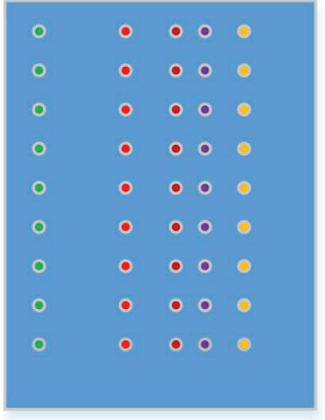

Figure 11: Schematic illustration on the service detector when using the FISLI as input for the LRSPE

Two additional wavelengths are introduced for the FM (Disco FISLI) phase in order to characterize the spectrometer also on the far wavelength end. Therefore a wavelength of $705 \mathrm{~nm}$ and $730 \mathrm{~nm}$ is added to each of the nine output channels. This results into the simultaneous imaging of 5x9 (45) spots onto the Flex CCD detector (Figure 12).

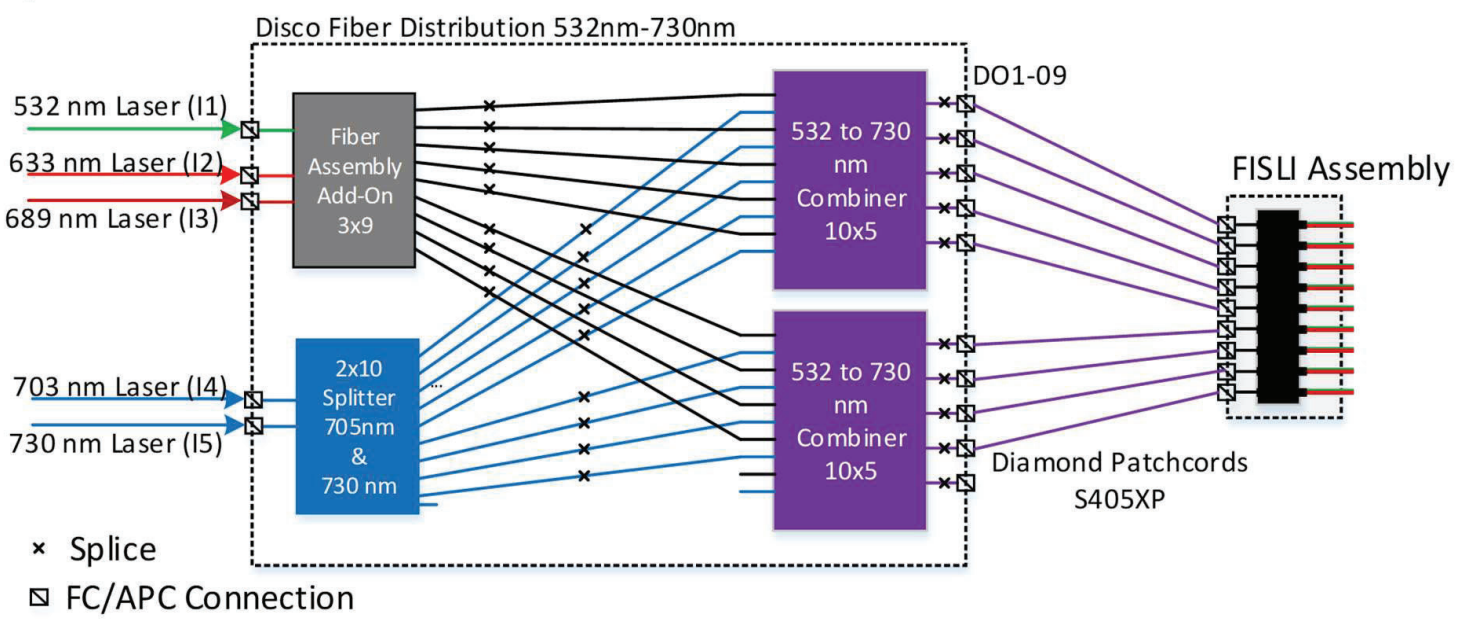

Figure 12: Disco FISLI fiber distribution principle in FM version.

In order to correct the positions of the field points on the service detector and to increase the accuracy of the smile and keystone evaluation, the relative position of the beam waist, in each fiber were characterized.

\section{CONCLUSION}

The BB campaign has proven that the theoretical developed alignment concept could be directly applied to the BB Instrument. The BB resulted compliant to all the optical performances. Some measured image quality parameters as the WFE, MTF and keystone resulted much better than required.

For Flight LRSPE the established updated alignment concept will be validated during the FM campaign. The necessary update of the different OGSE is already implemented. The flight optical and mechanical parts are currently in the manufacturing phase and some parts, as the flight lens and the flight flat mirror, are already in OHB. The assembly, alignment and testing is planned to be performed in 2021. The delivery of the flight LRSPE is foreseen for end of 2021. 


\section{REFERENCES}

[1] M. Drusch et al., "The fluorescence explorer mission concept—ESA's Earth Explorer 8," IEEE Trans. Geosci. Remote Sens. 55, 1273-1284 (2017).

[2] J. Nieke et al., "The Ocean and Land Colour Imager (OLCI) for the Sentinel 3 GMES Mission: status and first test results," Proc. SPIE 8528, 85280C (2012).

[3] P. Coppo et al., "SLSTR: a high dual scan temperature radiometer for sea and land surface monitoring from space," J. Mod. Opt. 57, 1815-1830 (2010).

[4] P. Coppo et al., "Sea and Land Surface Temperature Radiometer detection assembly design and performance," J. Appl. Remote Sens. 8, 084979 (2014).

[5] P. Coppo et al., "Fluorescence imaging spectrometer (FLORIS) for ESA FLEX mission, special issue "remote sensing of vegetation fluorescence and photosynthetic efficiency" (ISSN 2072-4292)," Remote Sens. 9, 649 (2017).

[6] P. Coppo et al.,"Instrument predevelopment activities for FLEX mission,” Opt. Eng. 58(7), 075102 (2019), doi: 10.1117/1.OE.58.7.075102.

[7] D. Malacara, Optical Shop Testing, Third Ed., J. Wiley \& Sons (2007).

[8] M. Kroneberger et al, "Scattering from reflective diffraction gratings: The challenges of measurement and verification", SPIE Frankfurt (2017) 